\title{
THE RADICAL EQUATION $P\left(A_{n}\right)=(P(A))_{n}$
}

\author{
R. E. PROPES
}

(Received 22nd June 1973, revised 12th November 1973)

The purpose of this paper is to impose conditions on a radical class $P$ so that the $P$-radical of the ring of $n \times n$-matrices over a ring $A$ is equal to the ring of $n \times n$ matrices over the ring $P(A)$. In (1), Amitsur gave such conditions, but with the stipulation that the radical class $P$ contained all zero-rings (rings in which all products are zero). In what follows, we shall be working within the class of associative rings.

We show that if $P$ is a radical class which is (right or left)-hereditary and (right or left)-strong, then $P$ has the property that the $P$-radical of the ring of $n \times n$-matrices over a ring $A$ is equal to the ring of $n \times n$-matrices over the ring $P(A)$.

Definition 1. Let $P$ be a radical class. A left ideal $I$ of a ring $A$ is called a $P$-left ideal of $A$ if $I$ is a $P$-ring, i.e. if $I \in P$. We define $P$-right ideals of $A$ analogously.

Definition 2. As defined in (2), a radical class $P$ is said to be left-strong in case $P(A)$ contains all $P$-left ideals of $A$ for each ring $A$. The concept rightstrong is defined analogously. A strong radical class is one which is both leftstrong and right-strong.

Definition 3. A radical class $P$ is said to be left-hereditary if each left ideal of a $P$-ring is also a $P$-ring. Right-hereditary radicals are defined analogously. An hereditary radical class is one for which each ideal of a $P$-ring is also a $P$-ring.

Remark. If $P$ is the Brown-McCoy radical, then $P$ is hereditary and satisfies the equation $P\left(A_{n}\right)=(P(A))_{n}$. However, from (2, Example 3), $P$ is neither leftstrong nor right-strong and is neither left-hereditary nor right-hereditary.

We shall employ the following notation throughout.

If $A$ is a ring and $n$ is a positive integer, $A_{n}$ denotes the ring of $n \times n$-matrices over $A$. For $i, j \in\{1,2, \ldots, n\}, A_{i j}$ denotes the subring of $A_{n}$ consisting of all matrices with elements from $A$ in the $(i, j)$-position and with 0 's elsewhere.

For $i \in\{1,2, \ldots, n\}$, we define $R_{i}$ as the right ideal $\sum_{j=1}^{n} A_{i j}$ of $A_{n}$, and we define $L_{i}$ as the left ideal $\sum_{k=1}^{n} A_{k i}$ of $A_{n}$. If $x \in A$ and $J$ is a non-empty subset of $\{1,2, \ldots, n\}$ with $i \in J$, then $B_{J}(i, x)$ denotes the $n \times n$-matrix with $x$ in the $(i, j)$-position for all $j \in J$ and with 0 's elsewhere. Then $B_{J(i)}=\bigcup_{x \in A} B_{J}(i, x)$ is a left-ideal of the ring $R_{i}$. Moreover, $A \cong B_{J(i)}$ under the obvious mapping. 
Theorem 1. Let $P$ be a radical class, let $A$ be a ring, and let $n$ be a positive integer. The following statements are equivalent.

(i) If $A \in P$, then $A_{n} \in P$.

(ii) $(P(A))_{n} \subseteq P\left(A_{n}\right)$.

Proof. Assume (i). Now $P(A) \in P$ so that by (i), $(P(A))_{n} \in P$. Hence $(P(A))_{n} \subseteq P\left(A_{n}\right)$. Next assume (ii). Now $A \in P$ implies $P(A)=A$ so that $A_{n}=(P(A))_{n}$. By (ii), $(P(A))_{n} \subseteq P\left(A_{n}\right)$. Whence $A_{n}=P\left(A_{n}\right)$ and $A_{n} \in P$.

Theorem 2. Let $P$ be a radical class, let $A$ be a ring, and let $n$ be a positive integer. The following statements are equivalent.

(i) If $A_{n} \in P$, then $A \in P$.

(ii) $P\left(A_{n}\right) \subseteq(P(A))_{n}$.

Proof. Assume (i). By Lemma 7 of Snider (4), $P\left(A_{n}\right)=I_{n}$ for some ideal I of $A$. From (i), we have $I \in P$. Hence $I \subseteq P(A)$ and so $P\left(A_{n}\right)=I_{n} \subseteq(P(A))_{n}$. Assume (ii). Now $A_{n} \in P$ implies $P\left(A_{n}\right)=A_{n}$. Thus by (ii), $A_{n}=P\left(A_{n}\right) \subseteq(P(A))_{n}$ and so $A_{n}=(P(A))_{n}$. Whence $P(A)=A$ and $A \in P$.

Theorem 3. Let $P$ be a strong radical class. Then $A \in P$ implies $A_{n} \in P$.

Proof. The theorem is evident for $n=1$. Thus, let $n>1$. Let $i \in\{1,2, \ldots, n\}$ be fixed, and let $j \in\{1,2, \ldots, n\}$ with $j \neq i$. Set $J=\{i, j\}$. Then since $A \in P$ and $A \cong B_{J(i)}$, we have $B_{J(l)} \in P$. Since $P$ is strong and since $B_{J(i)}$ is a left ideal of the ring $R_{i}$, we have that $B_{J(i)} \subseteq P\left(R_{i}\right)$. Setting $K=\{i\}$ we likewise obtain $B_{K(i)} \subseteq P\left(R_{i}\right)$. Hence $B_{J(i)}+B_{K(i)} \subseteq P\left(R_{i}\right)$. Since $j \neq i$, and $j$ was otherwise arbitrary, then $R_{i} \subseteq P\left(R_{i}\right)$, i.e. $R_{i} \in P$. Now $R_{i}$ is a $P$-right ideal of $A_{n}$ so that, since $P$ is strong, we must have $R_{i} \subseteq P\left(A_{n}\right)$. This being true for $i=1,2, \ldots, n$, we obtain $\sum_{i=1}^{n} R_{i} \subseteq P\left(A_{n}\right)$. Hence $A_{n}=P\left(A_{n}\right)$ and $A_{n} \in P$.

Theorem 4. If $P$ is a left-hereditary (or a right-hereditary) radical class, then $A_{n} \in P$ implies $A \in P$.

Proof. Let $P$ be a left-hereditary radical class, and let $A_{n} \in P$. Since $L_{1}$ is a left ideal of $A_{n}$, and since $P$ is left-hereditary, then $L_{1} \in P$. Now $A$ is a homomorphic image of $L_{1}$ under the mapping

$$
\left[\begin{array}{llr}
a_{11} & 0 \ldots \ldots . \\
a_{12} & 0 \ldots \ldots . \\
\vdots & \vdots & \vdots \\
\vdots & \vdots & \vdots \\
a_{1 n} & 0 \ldots \ldots .0
\end{array}\right] \mapsto a_{11} .
$$

Thus $A \in P$. The proof for right-hereditary radicals is dual.

The proof of the next theorem is facilitated by a proposition which is due to M. Jaegermann (3). If $A$ is a ring, $A^{+}$denotes the zero-ring on $A$, i.e. the additive group of $A$ with all products being 0 . 
Proposition. If $P$ is a hereditary and left-strong (right-strong) radical class, then $A \in P$ implies $A^{+} \in P$.

Theorem 5. If $P$ is a hereditary and left-strong (right-strong) radical class, then $A \in P$ implies $A_{n} \in P$.

Proof. Let $P$ be hereditary and left-strong, and let $A \in P$. From the proof of Theorem 3, the right ideal $R_{i}$ of $A_{n}$ belongs to $P$. By the Proposition, $R_{i}{ }^{+} \in P$. Since the zero-rings $R_{i}{ }^{+}$and $L_{i}{ }^{+}$are isomorphic by the matrix transpose function, we have $L_{i}{ }^{+} \in P$. Now $\sum_{j \neq i} A_{j i}$ is an ideal of $L_{i}{ }^{+}$and so belongs to $P$, since $P$ is hereditary. But $\sum_{j \neq i} A_{j i}$ is also an ideal of the ring $L_{i}$, and $L_{i} / \sum_{j \neq i} A_{j i} \cong A$. Since $A \in P$ and $\sum_{j \neq i} A_{j i} \in P$, then $L_{i} \in P$. Since $i$ was arbitrary, and $P$ is leftstrong, then $\sum_{i=1}^{n} L_{i}=A_{n} \in P$.

The proof for $P$ hereditary and right-strong is dual.

Theorem 6. If $P$ is a radical class which is (right or left)-hereditary and (right or left)-strong, then $P\left(A_{n}\right)=(P(A))_{n}$ for each ring $A$ and for each positive integer $n$.

Proof. Since $P$ is (right or left)-hereditary, then $P$ is hereditary. The proof now follows from Theorem 4 and Theorem 5 .

\section{REFERENCES}

(1) S. Amitsur, A general theory of radicals II, Amer. J. Math. 76 (1954), 100-125.

(2) N. Divinsky, J. KREMPA and A. SULINSKI, Strong radical properties of alternative and associative rings, J. Algebra 17 (1971), 369-388.

(3) M. Jaegermann, Morita contexts and radicals, Bull. Acad. Polon. Sci, Ser Sci. Math. Astronom. Phys. 20 (1972), 619-623.

(4) R. SNider, Lattices of radicals, Pacific J. Math. 40 (1972), 207-220.

The University of Wisconsin-MilwaUkeE

Milwaukee, Wisconsin 